\title{
28 Research Square \\ Total knee arthroplasty in advanced active tuberculous arthritis of the knee
}

Jingsheng Wang ( $\nabla$ wangjingshengwjs@163.com )

Yantai Affiliated Hospital of Binzhou Mecical University

Tingting Liu

Yangtai Affiliated Hospital of Binzhou Medical University

Apeng Zou

Yantai Affiliated Hospital of Binzhou Medical University

Qingpeng Shi

Yantai Affiliated Hospital of Binzhou Medical University

Baisui Zhou

Yantai Affiliated Hospital of Binzhou Medical University

Shanrun Zhao

Yantai Affiliated Hospital of Binzhou Medical University

\section{Research article}

Keywords: Tuberculosis, Total knee arthroplasty, Antitubercular therapy

Posted Date: April 1st, 2020

DOl: https://doi.org/10.21203/rs.3.rs-19831/v1

License: (c) (i) This work is licensed under a Creative Commons Attribution 4.0 International License.

Read Full License 


\section{Abstract}

Background: While studies have indicated that total knee arthroplasty (TKA) is effective to treat the patients with advanced tuberculosis (TB) of the knee joint a consensus of views regarding surgical timing, prosthesis selection, and peri-operative antitubercular therapy has not been reached. The purpose of this study was to evaluate the safety of TKA in the patients with advanced active tuberculous arthritis of the knee.

Methods: Eight patients with advanced active tuberculous arthritis of the knee were reviewed in this study from 2010 to 2017 . The diagnosis of each patient was confirmed by postoperative histopathology revealing granulomatous lesions with epithelioid histiocytes surrounded by lymphocytes and positive acid-fast staining. Antitubercular medications and TKA with primary prosthesis were performed in all patients with one-stage TKA in six and two-stage in two. Local symptoms, erythrocyte sedimentation rate (ESR) values were used for detecting the recurrence of tuberculosis. The hospital for special surgery knee score (HSS) system was used to evaluate the function outcomes of the involved knees.

Results: Within an average follow-up period of 48.8 months, no recurrence of tuberculous infection was found in any of the patients. The ESR became normal (below $20 \mathrm{~mm} / \mathrm{h}$ ) within six months after TKA in all patients. The average HSS score improved from $32.4 \pm 8.6(18-42)$ points preoperatively to $85.6 \pm 9.7(68-$ $94)$ points $1.5-2.0$ years after surgery $(p<0.001)$. All knees showed good integrity and no loosening of prosthesis was found.

Conclusions: TKA for advanced active tuberculosis of the knee is a safe procedure providing symptomatic relief and functional improvement. A long infection-free interval is not a prerequisite for TKA. Wide surgical debridement and adequate post-operative antitubercular chemotherapy are the mainstay to eradicate the infection.

\section{Background}

Tuberculosis (TB) is an ancient human disease caused by Mycobacterium tuberculosis and remains a major global health challenge with an estimated 10 million new TB cases worldwide in 2018 [1]. TB is a multi-systemic disease with a protean presentation although it mainly affects the lungs [2]. The rate of extra-pulmonary TB worldwide is about $20 \%-40 \%$. Of cases with extra-pulmonary TB, $10 \%-25 \%$ have musculoskeletal involvement [3]. The most commonly affected site of extra-pulmonary TB infection is the spine, followed by the hip, knee, and ankle/foot $[4,5]$. Due to its non-specific presentation TB of the knee is often reaches a very advanced state before a definite diagnosis is made, which then leads to a poor prognosis [6]. For the patients with end-stage joint destruction surgical intervention is frequently required to relieve pain and to restore mobility. Traditionally arthrodesis and total knee arthroplasty (TKA) are the main available options for orthopedic surgeons in the treatment of end-stage TB of the knee [7-13]. Arthrodesis may lead to decreased function in the affected limb and is associated with multiple complications such as nonunion and leg-length discrepancy although it can provide control of the 
infection and relief of pain $[7,8]$. In this realm, TKA can provide substantial benefits over arthrodesis, permitting the restoration of both function and mobility. By now there have been several reports on successful management of TB of the knee with TKA [9-14].

While TKA has clear benefits as a treatment modality, there is no consensus on the appropriate treatment regimens for patients with osteoarticular tuberculosis [5]. For the timing of surgery, some authors suggested that TB should be inactive for several years prior to performing a TKA, while others proposed a much shorter interval $[9,14,15]$. There are even a few articles on patients with active tuberculosis of the knee joint treated with TKA $[12,13,16]$. Additionally, when treating an active TB of the knee there is no consensus on whether to use a single- or two-stage TKA [5]. Therefore, the use of TKA in the face of an active tuberculous infection is still controversial and should be further investigated.

Based on previous experience on active tuberculosis of hip treated with total hip arthroplasty [17] we performed TKA procedures on eight knees with active tuberculosis. The aim of the present study was to evaluate our surgical protocols and clinical outcomes of TKA in patients suffering from advanced active tuberculous arthritis of the knee.

\section{Materials And Methods}

This study was approved by the review board of our hospital and the informed consent was acquired from every patients. From January 2010 to December 2017, eight patients with advanced active tuberculous arthritis of the knee were admitted to our institution. There were three men and five women with the mean age of 57.3 years ranging from 47 to 67 . The demographic details of all patients are shown in Table 1.The preoperative diagnosis of TB of the knee joint was based on past history, clinical symptoms (unilateral excruciating knee pain, joint swelling, and disability) and imaging studies (the Phemister triad: juxta-articular osteoporosis, peripherally located osseous erosions, and gradual narrowing of the joint space) (Fig. 1a, b). Clinical suspicion and a well-documented history were the most important steps in diagnosis of TB of the knee. Magnetic resonance imaging (MRI) was carried out to define the extent of tuberculous infection. Computed tomography (CT) of lung and spine were obtained to rule out other active foci of concomitant tuberculous infection. Popliteal abscess was found in one patient (Case 5). One of them had a history of previous pulmonary tuberculosis and suffered from a recent onset discharging sinus tract in his right knee (Case7). Positive preoperative symptoms and elevated erythrocyte sedimentation rate (ESR) were used to evaluate preoperative activation and postoperative recurrence of TB. All patients were free from contraindications caused by other systems. The hospital for special surgery knee score (HSS) system was used to evaluate the function outcomes of the knees. The preoperative HSS scores were acquired at admission. 
Table 1

Clinical data of 8 patients

\begin{tabular}{|c|c|c|c|c|c|c|c|c|}
\hline Patients & Gender & Age & $\begin{array}{l}\text { Pre-anti-TB } \\
\text { /Pre-operation } \\
\text { ESR(mm/h) }\end{array}$ & \multicolumn{2}{|c|}{$\begin{array}{l}\text { Antitubercular } \\
\text { therapy }\end{array}$} & $\begin{array}{l}\text { follow-up } \\
\text { period } \\
\text { (months) }\end{array}$ & \multicolumn{2}{|c|}{ HSS } \\
\hline $\begin{array}{l}\text { Pre- } \\
\text { operation }\end{array}$ & $\begin{array}{l}\text { follow- } \\
\text { up }\end{array}$ & $\begin{array}{l}\text { Pre- } \\
\text { operation } \\
\text { (days ) }\end{array}$ & $\begin{array}{l}\text { Post-operation } \\
\text { (months) }\end{array}$ & & & & & \\
\hline 1 & $F$ & 47 & $114 / 82$ & 24 & 12 & 78 & 18 & 92 \\
\hline 2 & $F$ & 62 & $54 / 46$ & 18 & 12 & 71 & 26 & 85 \\
\hline 3 & $M$ & 53 & $69 / 51$ & 15 & 12 & 63 & 42 & 89 \\
\hline 4 & $\mathrm{~F}$ & 59 & $32 / 26$ & 14 & 12 & 60 & 38 & 94 \\
\hline 5 & $F$ & 67 & $48 / 44$ & 94 & 12 & 38 & 26 & 68 \\
\hline 6 & $M$ & 56 & $70 / 49$ & 17 & 12 & 26 & 31 & 94 \\
\hline 7 & $M$ & 65 & $90 / 67$ & 110 & 12 & 30 & 36 & 74 \\
\hline 8 & $\mathrm{~F}$ & 49 & $72 / 37$ & 14 & 12 & 24 & 42 & 90 \\
\hline
\end{tabular}

Preoperative antitubercular drugs were administered once TB was diagnosed clinically. Preoperative drugs included isoniazid (300 mg, once daily), rifampicin (450 mg, once daily), ethambutol (750 mg, once daily), and streptomycin $(750 \mathrm{mg} / \mathrm{d}$, once daily $\times 3$ months). Two patients were treated with two-stage TKA. The goal of the first stage of surgery was to debride the popliteal tuberculous abscess in one patient (Case 5) and eliminate the sinus tract for fear of mixed infection in another (Case 7). The final stage of surgery was not performed until the incisions healed without complications. This interval was usually about 3 months. The other six patients were treated with one-stage TKA. For these patients the preoperative antitubercular medication was given for at least 2 weeks to make the ESR decrease more than $20 \%$ or below $50 \mathrm{~mm} / \mathrm{h}$. All TKA procedures were performed by a senior surgeon, using continuous epidural anesthesia and via a medial parapatellar approach. The surgical operation was in consistent with the common practices with inflammatory synovium and caseous substance being debrided thoroughly. Medical pulse irrigator was used in all patients after osteotomy and debridement were completed. Primary cemented posterior-stabilized prostheses (Waldemar Link, Hamburg, Germany) were used in all cases. Local isoniazid (300 mg) and streptomycin $(250 \mathrm{mg}$ ) were administered in joint cavity in all cases before the incisions were closed. Drainage was performed in all cases and removed 24-48 hours after surgery. The resected tissues were sent for histopathologic examination, acid-fast staining and tuberculosis culture for further diagnosis. 
Antitubercular drugs were continued for 12 months after surgery in all patients. Cefuroxime was used for 72 hours to prevent additional infection postoperatively. Enoxaparin was used for two weeks to prevent deep venous thromboembolism. ESR was examined every two months postoperatively until it was below $20 \mathrm{~mm} / \mathrm{h}$. Follow-up was performed every two months within the first 6 months and once per year. The postoperative HSS scores were acquired 1.5-2 years after surgery. Re-aggravated knee pain, joint swelling, and disability were considered as possible recurrence of TB during follow-up and further investigation must be performed.

Statistical analysis was evaluated by paired t tests using SPSS 19.0 statistical software (SPSS Inc., Chicago, IL, USA). Significance was defined as $\mathrm{P}<0.05$. Preoperative and postoperative HSS scores were compared.

\section{Results}

Intraoperatively synovial hyperplasia edema and worm-eaten damage of cartilage and subchondral bone were found in all patients. Caseous tissue was seen in five patients (Fig. 2). An intraoperative complication happened in one patient (Case 5) whose tibia end of the patellar tendon was torn from the tubercle of tibia due to stiffness of the involved knee. The patellar tendon was fixed by two anchors. A semi-confined brace was used for six weeks after surgery. No postoperative complications such as pyogenic infection, deep vein thrombosis were observed during the follow-ups. Histopathologically, TB was confirmed by typical granulomatous lesions including epithelioid histiocytes surrounded by lymphocytes in all patients. Acid-fast staining bacilli were detected in all specimens and culture was negative in all patients. The postoperative ESR decreased below $20 \mathrm{~mm} / \mathrm{h}$ within six months in all patients. Within an average follow-up period of $48.8(24-78)$ months no recurrence of TB was found. The average HSS score improved from $32.4 \pm 8.6(18-42)$ points preoperatively to $85.6 \pm 9.7(68-94)$ points 1.5-2.0 years after surgery $(p<0.001)$ (Table 1$)$. At the last follow-up, all knees showed good integrity and all patients could ambulate without walking aids, and radiographs did not reveal any loosening of the prostheses (Fig. 3).

\section{Discussion}

Due to lack of specific presentation most patients with TB of the knee are in severe disability with joint destruction and loss of articular congruity when they are diagnosed as TB. While several studies have indicated that TKA is effective to treat the patients with advanced TB of the knee joint a consensus of views regarding surgical timing, prosthesis selection, and peri-operative antitubercular therapy has not been reached $[11-14,16,18]$. The results of the present study revealed that 2 to 4 weeks of preoperative antitubercular therapy was enough for the patient with advanced active TB of the knee. We recommend one-stage TKA for the patients with advanced active TB of the knee unless there is a discharging sinus tract or abscess that cannot be debrided during the surgery for TKA. In line with previous studies we recommend a long period of postoperative antitubercular therapy which should be at least 12 months after surgery to reduce the rate of local recurrence of TB $[11-14,16,18]$. 
TKA can provide the patients with quiescent TB of the knee with an excellent function, but joint replacement for active tuberculous infection was opposed by most authors because they believed the risks were too high [20]. Other authors recommend a long interval between the treatment of the active infection and arthroplasty $[11,12,14,18]$. They believe that the prolonged preoperative antitubercular therapy could reduce the reactivation risk of TB infection. However, the optimal timing for TKA in patients with TB of the knee joint is still controversy. Su et al [11] reported sixteen cases of tuberculosis of the knee treated with TKA. Eight cases were treated with antitubercular therapy for 2 ( 3 cases) to 12 ( 5 cases) months before and 1 year after arthroplasty. Another 8 cases were not diagnosed primarily and therefore received only postoperative antitubercular therapy. Five cases had a recurrence of TB. Four of these 5 cases did not receive preoperative antitubercular treatment. The last case of recurrent infection occurred in a patient who had received longstanding corticosteroid therapy. They suggested that effective preoperative and postoperative antitubercular chemotherapy were mandatory for the prevention of tuberculous reactivation after TKA. Oztürkmen et al. [12] reported twelve patients with recent onset tuberculous arthritis of the knee. Two-stage TKA was performed in all patients with 7 primary prostheses and 5 revisions. The time interval between the first and the second stage was not more than 6 months. All patients were given postoperative antitubercular treatment for more than 1 year, and within an average follow-up period of $6.1 \pm 1.8$ years, no reactivation of tuberculous infection was found. Kim et al. [13] performed TKA in 19 patients (22 knees) with tuberculous arthritis of the knee. The interval between the subsidence of the signs of tuberculous infection and arthroplasty ranged from 3 months to 5 years. They found 3 recurrent cases during the follow-up. Habaxi et al [14] analyzed 10 patients with active tuberculosis of the knee. One-stage TKA was performed in all cases. The patients were given preoperative antitubercular treatment for 2-4 weeks and more than 1 year postoperatively. They had one recurrent case. Our study is in line with Habaxi et al. In our series, preoperative antitubercular therapy was administered for 2-4 weeks in six patients and no recurrence was found during follow-up. Two other cases received antitubercular treatment for more than 3 months because popliteal abscess and discharging sinus tract had to be eliminated before TKA. No recurrence of TB was found in these two cases. From the results of our study and reviews of previous studies we find that the recurrence of TB after TKA is not related to the interval between initiation of preoperative antitubercular treatment and arthroplasty. The recurrence of TB after TKA may occur even when TB is quiescent for several years [13, 19] while patients without preoperative antitubercular treatment may be free from recurrence $[11,16,21]$. We recommend short-term preoperative antitubercular treatment so as to shorten the course of treatment of TB. In our opinion 2-4 weeks of preoperative antitubercular treatment is enough before TKA if there are no special conditions such as sinus tract or extra-articular abscess.

The reason that we recommend short-term preoperative antitubercular treatment lies in that $\mathrm{M}$. tuberculosis has specific behavioral characteristics differ from pyogenic bacteria [22]. M. tuberculosis reproduces slowly and has little tendency to adhere to implants. In vitro studies also demonstrated that M. tuberculosis has little or no biofilm formation making it susceptible to antitubercular therapy [23]. So antitubercular drugs can eliminate M. tuberculosis efficiently even with implants existed. Even if TB is 
reactivated, the disease can be controlled by antitubercular drugs alone or in combination with debridement without removal of the prosthesis in most cases $[11,13,19,24]$.

Complete debridement of the infected tissues is the mainstay of the treatment to lower the potential risk of reactivation of TB [11-14]. However, it's difficult to cure tuberculous infection using debridement in combination with antitubercular drugs. The risk of recurrence is high according to previous reports [13, 14]. Moreover, the patients have to suffer from disability of the knee before arthroplasty because the joint surface would be more ragged after debridement. Additionally it's difficult to perform thorough debridement in that surgeons have to keep bone tissues as more as possible for fear of massive bone defect. The situation is different in TKA because the infected tissues are eliminated to the most extent due to extensive intraoperative exposure and bone osteotomy. Intraoperative pulsed irrigation may further reduce $M$. tuberculosis located in trabecular space.

Some authors recommended two-stage surgery in patients with active tuberculous arthritis $[12,18,25]$. However, two-stage TKA may cause so much loss of bone that revision prostheses have to be needed in some cases which may impair the function of the knee and the life of the prosthesis [12, 25]. In our series, two-stage TKA was performed in two cases due to popliteal abscess or discharging sinus tract. In fact, the surgical procedures were more difficult than usual due to the stiffness caused by less movement of the knee after the first-stage surgery. At the last follow-up, although no recurrence of TB was found the function of the knee with two-stage TKA was poorer than that with one-stage surgery (Table 1). In our opinion, two-stage TKA for active tuberculosis of the knee is not recommended unless there is discharging sinus tract or extra-articular abscess unable to remove during TKA.

As for postoperative antitubercular therapy our opinion is in line with others $[11-14,16,18]$. We recommend a long-term postoperative antitubercular therapy for at least 12 months. Once recurrence of TB is detected a longer antitubercular course is recommended until TB is cured. Debridement with or without removal of implants may be needed sometimes.

Several authors considered the level of ESR as an indicator of the efficacy of antitubercular drugs $[11,18]$. We agree with this point. In our study TKA was not performed until the ESR decreased more than $20 \%$ or below $50 \mathrm{~mm} / \mathrm{h}$. Normal ESR levels are not prerequisite for TKA because the signs of active infection (persistent pain, effusion in the joint, and positive radiographic features) may exist despite the fact that laboratory tests show no evidence of active infection [13]. The present study reveals that TKA for patients with active TB of the knee is a safe procedure even though the ESR level is higher than normal.

\section{Conclusions}

TKA for advanced active tuberculosis of the knee is a safe procedure providing symptomatic relief and functional improvement. A long infection-free interval is not a prerequisite for TKA. Wide surgical debridement and adequate postoperative antitubercular chemotherapy are the mainstay to eradicate the infection and reduce the risk of reactivation of TB. 


\section{Abbreviations}

TKA: total knee arthroplasty; TB: tuberculosis; ESR: erythrocyte sedimentation rate; HSS: The hospital for special surgery knee score

\section{Declarations}

\section{Ethics approval and consent to participate}

This study was approved by the review board of our hospital and the informed consent was acquired from every patients. Consent for publication

Not applicable.

\section{Availability of data and materials}

Yes, data and material were available, not been published, and is not under consideration elsewhere.

\section{Competing interests}

The authors declare that they have no competing interests.

\section{Funding}

The study did not receive any specific grant from funding agencies in the public, commercial or not-forprofit sectors.

\section{Authors' contributions}

All authors participated in the design, interpretation of the studies, analysis of the data, and review of the manuscript. All authors read and approved the final manuscript.

\section{Acknowledgements}

Not applicable.

\section{References}

1. For the WHO recommendations, see https://www.who.int/tb/global-report-2019.

2. Adigun R, Singh R. Tuberculosis. [Updated 2019 Feb 6]. In: StatPearls [Internet]. Treasure Island (FL): StatPearls Publishing; 2020 Jan-. Available from: https://www.ncbi.nlm.nih.gov/books/NBK441916/

3. Held MFG, Hoppe S, Laubscher M, et al. Epidemiology of Musculoskeletal Tuberculosis in an Area with High Disease Prevalence. Asian Spine J. 2017; 11(3):405-411. 
4. Johansen IS, Nielsen SL, Hove M, et al. Characteris-tics and clinical outcome of bone and joint tuberculo-sis from 1994 to 2011: a retrospective register-based study in Denmark. Clin Infect Dis. $2015 ; 61: 554-62$.

5. Qian Y, Han Q, Liu W, Yuan WE, Fan C. Characteristics and management of bone and joint tuberculosis in native and migrant population in Shanghai during 2011 to 2015 . BMC Infect Dis.2018; 18(1):543.

6. Sultan AA, Cantrell WA, Rose E, et al. Total knee arthroplasty in the face of a previous tuberculosis infection of the knee: what do we know in 2018? Expert Rev Med Devices. 2018; 15(10): 717-724.

7. Tang X, Zhu J, Li Q, Chen G, Fu W, Li J. Knee arthrodesis using a unilateral external fixator combined with crossed cannulated screws for the treatment of end-stage tuberculosis of the knee. BMC Musculoskelet Disord. 2015; 16:197.

8. Lim HC, Bae JH, Hur CR, Oh JK, Han SH. Arthrodesis of the knee using cannulated screws. J Bone Joint Surg Br. 2009; 91(2):180-184

9. Tuli, SM. General Principles of Osteoarticular Tuberculosis.Clin Orthop Relat Res. 2002; 398: 11-19.

10. Bae DK, Yoon KH, Kim HS, Song SJ. Total knee arthroplasty in stiff knees after previous infection. J Bone Joint Surg Br. 2005; 87(3):333-336.

11. Su JY, Huang TL, Lin JY. Total knee arthroplasty in tuberculous arthritis. Clin Orthop Relat Res.1996; 323(2):181-187.

12. Oztürkmen $\mathrm{Y}$, Uzümcügil $\mathrm{O}$, Karamehmetoğlu $\mathrm{M}$, Leblebici C,Caniklioğlu M. Total knee arthroplasty for the management of joint destruction in tuberculous arthritis. Knee Surg Sports Traumatol Arthrosc. 2014; 22 (5): 1076-1083.

13. Kim YH. Total knee arthroplasty for tuberculous arthritis. J. Bone Joint Surg. Am.1988; 70:13221330.

14. Habaxi KK, Wang L, Miao XG, et al. Total knee arthroplasty treatment of active tuberculosis of the knee: a review of 10 cases. Eur Rev Med Pharmacol Sci. 2014; 18 (23): 3587-3592.

15. Watts HG, Lifeso RM. Tuberculosis of bones and joints. J. Bone Joint Surg. Am.1996; 78:288-298.

16. Gale DW, Hading ML. Total knee arthroplasty in the presence of active tuberculosis. J Bone Joint Surg Br. 1991; 73(6): 1006-1007.

17. Wang $Y$, Wang J, Xu Z, Li Y, Wang $\mathrm{H}$. Total hip arthroplasty for active tuberculosis of the hip. Int Orthop. 2010; 34(8): 1111-1114.

18. Zeng M, Xie J, Wang L, Hu Y.Total knee arthroplasty in advanced tuberculous arthritis of the knee. Int Orthop. 2016; 40(7):1433-1439.

19. Eskola A, Santavirta S, Konttinen YT, Tallroth K, Lindholm ST. Arthroplasty for old tuberculosis of the knee. J Bone Joint Surg Br. 1988; 70(5):767-769.

20. Babhulkar S, Pande S. Tuberculosis of the hip. Clin Orthop Relat Res 2002; 398:93-99.

21. Besser MI. Total knee replacement in unsuspected tuberculosis of the joint.Br Med J. 1980; 280(6229):1434. 
22. Moon MS. Tuberculosis of spine: current views in diagnosis and management. Asian Spine J. 2014; 8(1):97-111.

23. Ha KY, Chung YG, Ryoo SJ. Adherence and biofilm formation of Staphylococcus epidermidis and Mycobacterium tuberculosis on various spinal implants. Spine. 2005; 30(1)38-43.

24. Haan JD, Vreeling AWJ, Hellemondt GGV. Reactivation of ancient joint tuberculosis of the knee following total knee arthroplasty after 61 years: a case report. Knee. 2008; 15(4):336-338.

25. Leclere LE, Sechriest $\mathbb{V F}$, Holley KG, Tsukayama DT. Tuberculous arthritis of the knee treated with two-stage total knee arthroplasty. A case report. J Bone Joint Surg Am. 2009; 91(1):186-191.

\section{Figures}

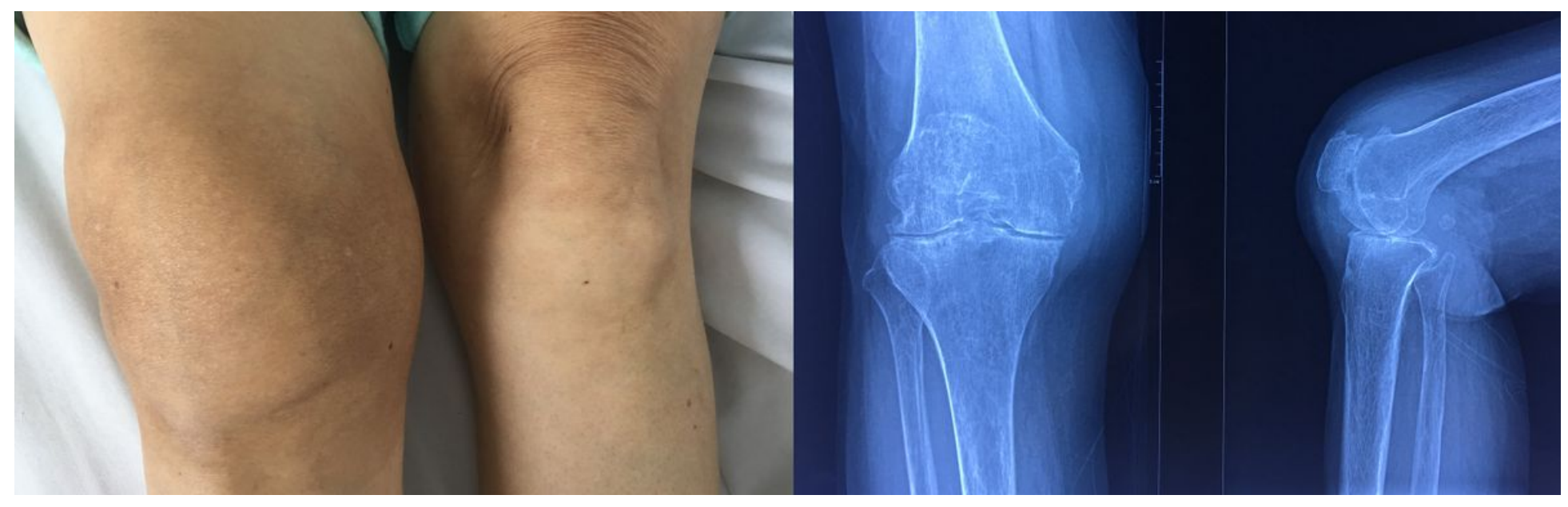

Figure 1

A 62 years old female, suffering from right knee pain for 1 year, cannot walk for 2 months. a: obvious swelling in her right knee; $b$ : the Phemister triad was shown on her anteroposterior and lateral radiographs. 


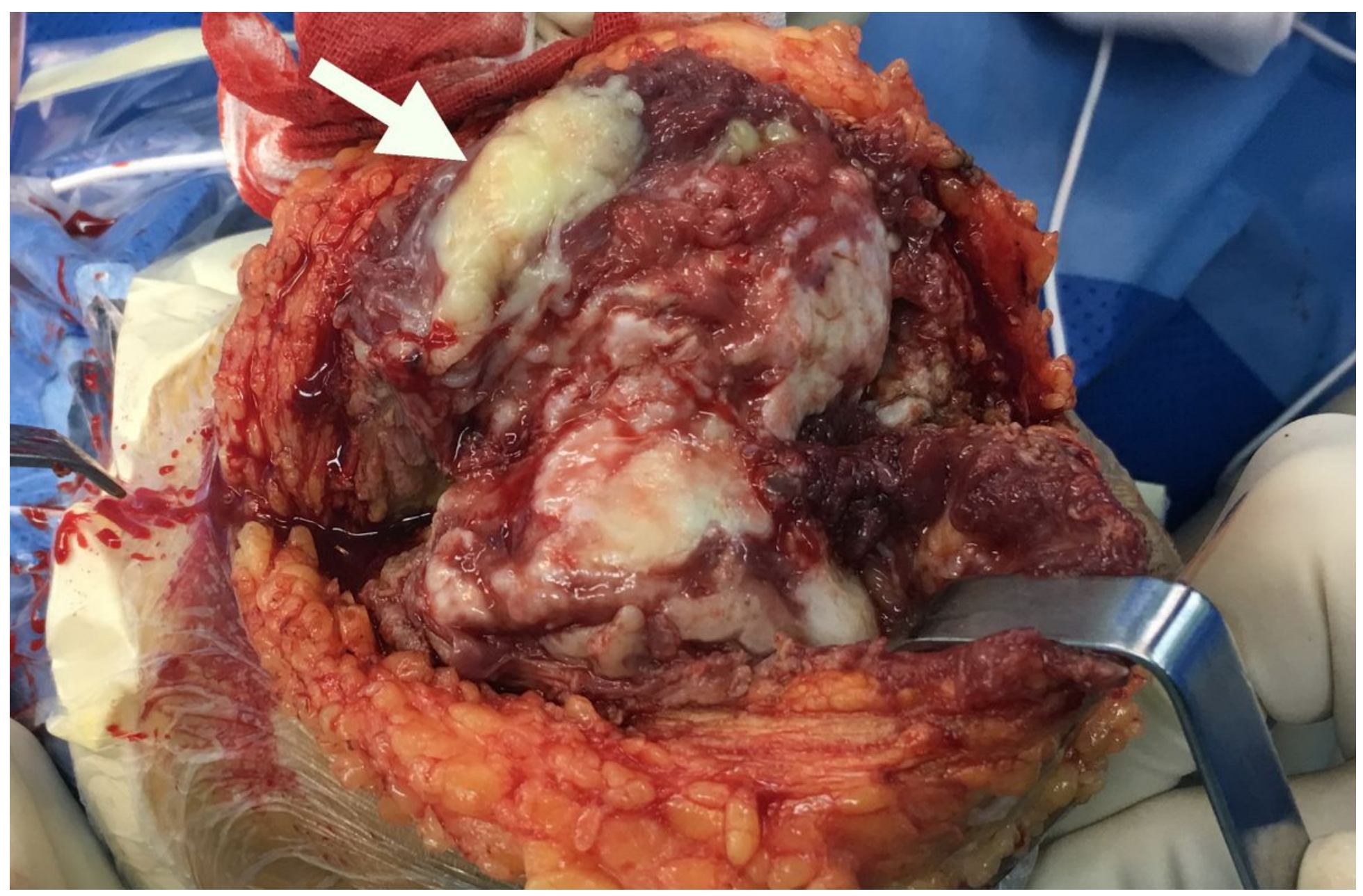

Figure 2

Synovial hyperplasia edema and worm-eaten damage of cartilage and subchondral bone were found intraoperatively. Caseous tissue was also obvious (white arrow). 


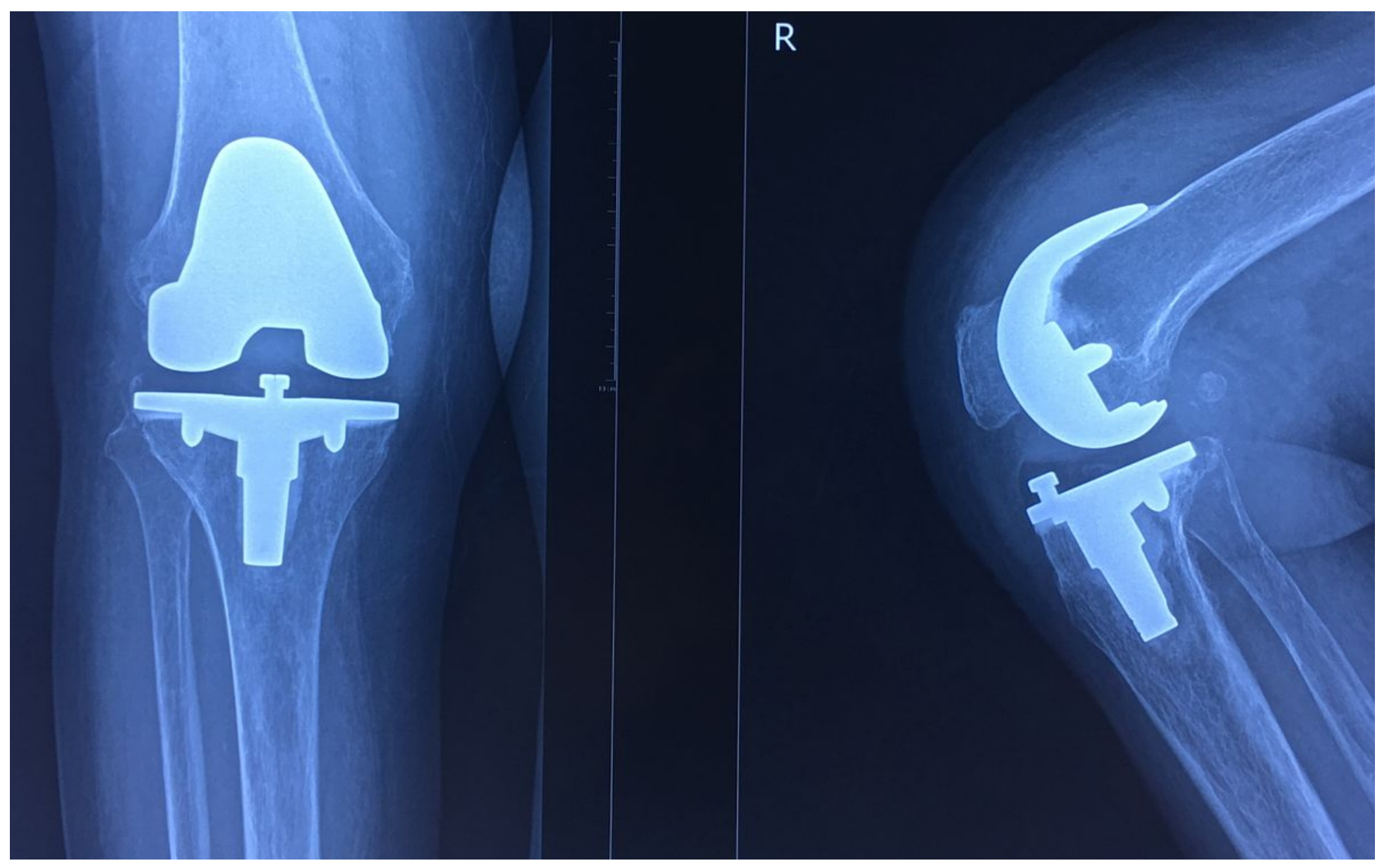

\section{Figure 3}

Anteroposterior and lateral radiographs 24 months after surgery, showing no signs of loosening of prosthesis. 